approaches. Included in training are marital and family contract therapy for marital disharmony, treatment of mothers who abuse their children, and management of other behavioural problems.

Nurse-therapy training programmes yield a valuable clinical service to their training hospitals. During the first two Maudsley training programmes and secondment elsewhere, ten trainee nursetherapists completed treatment of 387 patients. Lasting improvement was obtained in adult neurotic problems such as phobias, obsessive-compulsive rituals, social skills deficits, sexual dysfunction, sexual deviation, and habit disorders. Treatment was brief, in a mean of 11 to 12 sessions. Improvement was of the same order as that obtained with comparable treatments and measurements by psychiatrists, psychologists and medical students in Britain and Canada. Results from the second Maudsley training programme were at least as good as in the first one, and early outcome from the third programme is equally satisfactory.

When nurse-therapists move into service settings, responsible consultants are often too busy to carry out assessment for suitability for treatment, and this task then becomes the responsibility of the nursetherapist. A controlled comparison of their decisions about patients' management in selected adult neurotic patients showed that decisions were similar to those made by a psychiatrist.

Nurse-therapists have also shown promise in general practice settings. Several attachments worked well. An advantage of general practice rather than hospital settings is that they shorten the chain of referral before treatment. Patients can thus obtain treatment earlier. Another gain is that many patients may accept treatment who would refuse it in a psychiatric setting because of the distance from their homes or the stigma attached to the label of being a psychiatric patient.

An obvious question is what happens to nursetherapists after they leave the course. Of the first fourteen graduates from the Maudsley and Graylingwell courses, all but one subsequently worked as a nurse-therapist in some capacity. One has become a nurse-tutor and another a clinical nurse-teacher to the existing nurse-therapy courses, while another is a clinical teacher running a JBCNS course in behaviour modification for the mentally handicapped. A nursing officer has been appointed by the Sheffield AHA with nurse-therapy as a major component in a job description, thus providing a precedent for nursetherapists to gain promotion in the nursing hierarchy on clinical grounds rather than for the more usual reasons for advancement through administration or teaching.

Nurse-therapy is generally a satisfying role for nurses who have gone through the course, and can help increase the attractiveness of nursing as a profession. At the same time it increases the availability of behavioural psychotherapy to patients who can benefit from it but formerly were often denied it for lack of trained personnel. The training of nurse-therapists allows the expansion of behavioural services at a cost lower than might otherwise be the case. It also frees psychiatrists and psychologists to use their time more as consultants in deed as well as in name. Further potential of this model of service delivery is under exploration.

\title{
GLINICAL TRIAL OF LITHIUM AND AMITRIPTYLINE IN RECURRENT DEPRESSIVE ILLNESS
}

The Medical Research Council is currently engaged in a clinical trial comparing the effects of lithium and amitriptyline in recurrent unipolar depressive illness. Blood levels of amitriptyline and of lithium are being monitored to obtain optimal conditions for both drugs.

Eight hospitals throughout the UK are currently involved in the project and the major part of the trial should be completed by the end of this year provided that a satisfactory number of patients come into the trial. Psychiatrists have already been circulated by the Department of Health and Social Security and the Scottish Home and
Health Department drawing their attention to it. Clearly both amitriptyline and lithium are potentially toxic preparations, although both have proved valuable in the prophylaxis of recurrent depressive illness. It would be to the advantage of patients and to the efficiency of the National Health Service if an early result from this trial could be obtained.

The UK Trial Coordinator, from whom further information is obtainable, is Dr Iain Glen, MRC Brain Metabolism Unit, Thomas Clouston Clinic, 153 Morningside Drive, Edinburgh, EH 10 5LG, telephone number (031) 4472011 , extension 530. 